\title{
On the well-posedness of the multi-dimensional Roe-Liu-Vinokur linearization for residual distribution schemes
}

Jesús Garicano-Mena a,* Gérard Degrez ${ }^{\mathrm{b}, \mathrm{c}}$

\section{Introduction}

In reference [1], Liu and Vinokur proposed an strategy to derive a Roe-like linearization for flows in thermo-chemical non-equilibrium (TCNEQ). In the context of approximated Riemann solvers, being able to determine a Roe-like averaged state $\bar{Z}_{\text {avg }}$ respecting property $U$ (see [2]) guarantees that a discrete linearized description of the Riemann problem provides a solution consistent with that of the original non-linear problem, i.e., the numerical algorithm can - in the words of Roe "recognize a slock wave".

Litu and Vinokur's accomplishment was to offer Roe-like averaged states $\bar{Z}_{\text {avg }}$ under conditions for which the existence of such averages was not guaranteed, namely for complex, highly non-linear thermodynamic models. References [3,4] illustrate the usage of liu and Vinokur's generalized Roe average for combustion applications.

Note that most applications resorting to the generalized Roe average apply it on a dimension-by-dimension basis. Seeking to take advantage of the multi-dimensional upwind residual distribution algorithms in [5], Degrez and van der Weide devised an strategy to extend the Roe-Liu-Vinokur ( $R L V$ ) linearization to the multi-dimensional case. In [6] we revisited the linearization procedure, describing it in detail. In this note we investigate the conditions under which the linearization is well defined. 
In attention to the limited space in Short Notes, the goveming equations and their discretized version are presented very briefly in section 2 . The reader interested will find the complete details on the application of the Residual Distribution (RD) method to the $n_{s}$ species, two-temperature TCNEQ model in reference [7]. Next, the multi-dimensional linearization procedure is described in section 3; an application case of the multi-dimensional linearization is also presented, and several of its shortcomings are identified. In section 4 we provide an explanation for this behavior. We finish the Short Note with the Conclusions, including a series of recommendations for the simulation of shocked hypersonic flow fields with Residual Distribution schemes.

\section{The governing equations and its RD-discretized counterpart}

\subsection{Govening equations}

In this work inviscid NEQ flows including vibrational excitation and chemical reaction processes, described with a $n_{5}$ species (with $n_{S} \geq 2$ ), two-temperature model are considered. The system of conservation equations for such situation read in compact vector form as:

$$
\frac{\partial \vec{U}}{\partial t}+\nabla \cdot \overline{\bar{F}}^{c v}=\vec{S}
$$

Here $\bar{U}$ are the conserved variables, tensor $\overline{\bar{F}}^{c}$ collects the convective fluxes and vector $\vec{S}$ contains the chemical and internal energy modes source terms. For a TCNEQ flow the vector of conserved variables is:

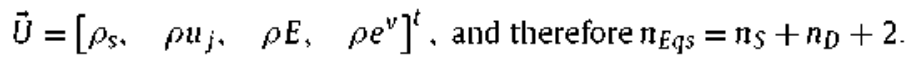

In system above, $\rho_{s}$ stands for the density of the $s$-th species, while $\rho \vec{u}, \rho E$ and $\rho e^{v}$ are respectively the momentum, the total energy and the electronic-vibrational energy per unit volume. Additionally, $p$ is the pressure exerted by the mixture and $H$ is the specific total enthalpy. The convective tensor is defined as $\overline{\bar{F}}^{c v}=\bar{F}_{j}^{c v}+\bar{e}_{j}^{t}$ for $j \in\left\{x_{1} \ldots, x_{n_{0}}\right\}$ (Einstein convention applies); the source term is $\vec{S}=\left[\dot{\omega}_{1}, \ldots, \dot{\omega}_{n_{s}}, \overline{0}_{1 \backslash \pi_{0}}, 0, \Omega^{v}\right]^{t}$. Introduction of the advective Jacobian matrices $A_{j}^{c v}$ leads to the quasj-linear form of the system of equations:

$$
\frac{\partial \vec{U}}{\partial t}+\underbrace{\frac{\partial \vec{F}_{j}^{c \nu}}{\partial \vec{U}}}_{A_{i}^{c \nu}} \cdot \frac{\partial \vec{U}}{\partial x_{j}}=\vec{S}
$$

Note that the vectors/matrices employed fulfill $\bar{U}, \bar{F}_{j}^{c v}, \vec{S} \in \mathbb{R}^{n n_{E s}}$ and $\vec{A}_{j}^{c v} \in \mathbb{R}^{n_{E q s} / n_{E q s}} ;\left\{\vec{e}_{j}\right\}$ is the canonical basis for $\mathbb{R}^{n_{D}}$. This work is concerned with the possibility of identifying a favorable state-averaging operator for which the evaluation of $\bar{A}_{j}^{c v}$ guarantees conservation at the discrete level.

The specific form of the mass production $\dot{\omega}_{s}$ and the energy relaxation $\Omega^{v}$ terms is not relevant for the linearization procedure; the interested reader can consult [8]. Details on initial/boundary conditions can be found in [9,10]; whereas the calculation of all chemistry, thermodynamics and energy transfer properties is described in [11,12].

At the equation of state level, the ideal gas law for a thermally perfect gas ( $P G$ ) applies to each of the components of the gas mixture, which exert a partial pressure given by $p_{s}=R / M_{s} \rho_{s} T$. Here, $R$ is the universal gas constant $R=8314.4 \mathrm{~J} / \mathrm{kkmol}$ and $M_{s}$ the $s$-th species molecular weight (in $\mathrm{k} / \mathrm{kmmol}$ ). The total pressure of the mixture is simply given by $p=\sum_{s=1}^{\prime \prime s} p_{s}$.

Specific enthalpy $H$ relates to pressure as $H=E+p / \rho$. Total energy $\rho E$ and pressure $p$ of the mixture can be further related if one realizes that $\rho E$ includes contributions from the translational-rotational modes, from the vibrational-electronic modes and from the kinetic energy, as in $\rho E=\rho e^{t t}+\rho e^{v}+\frac{1}{2} \rho \bar{u}^{t}$. $\bar{u}$. Each of these gathers in turn contributions from the different species in the mixture:

$$
\rho e^{t r}=\sum_{s=1}^{n_{s}} \rho_{s} e_{s}^{t r}, \rho e^{v}=\sum_{s=1}^{n_{s}} \rho_{s} e_{s}^{v}, \frac{1}{2} \rho \bar{t}^{r} \cdot \bar{t}=\sum_{s=1}^{n_{s}} \rho_{s} \frac{\|\vec{t}\|^{2}}{2} .
$$

The $s$-th species translational-rotational energy is $\varepsilon_{s}^{t r}=\int_{T_{0}}^{T} C_{v s}^{t r} d \tau+h_{s}^{0} ; h_{s}^{0}$ is the formation enthalpy of the species at the reference temperature $T^{0}$ and $C_{v, s}^{t r}$ is the translational-rotational specific heat at constant volume. Since the components of the mixture behave as calorically perfect gases, $C_{v, s}^{t r}=\frac{3}{2} R_{s}$ for monoatomic species and $\frac{5}{2} R_{s}$ for diatomic molecules.

The expression for the $s$-th species vibrational energy $e_{s}^{v}$ assumes that the internal quantum states are populated according to a Boltzmann distribution. Therefore $e_{s}^{v}=0$ for atomic species and $e_{s}^{v}=\frac{R}{M_{s}} \frac{\theta_{s}^{v}}{e_{s}^{v} i T^{v}-1}$ for diatomic molecules; $\theta_{s}^{v}$ is a characteristic vibrational temperature for the s-th species. 
Differentiation of the total pressure equation and algebraic manipulation, see Ref. [1], leads to:

$$
\mathrm{d} p=\sum_{s=1}^{n_{s}} \gamma_{s} \mathrm{~d} \rho_{s}+\beta \mathrm{d} \rho e^{\mathrm{tr}} .
$$

Terms $\gamma_{s}$ and $\beta$ in Eq. (4) stand respectively for the partial derivatives of pressure with respect to the translational-rotational energy and the species densities; they are given by:

$$
\beta=\frac{\partial p}{\partial \rho e^{t r}}=\frac{\sum_{s=1}^{n_{s}} y_{s}{ }^{R / M_{s}}}{\sum_{s=1}^{I_{S}} y_{s} C_{V, s}^{t r}} \text {, and } \gamma_{s}=\frac{\partial p}{\partial \rho_{s}}={ }^{R / M_{s}} T-\beta e_{s}^{t r} \text {. where } y_{s} \equiv \rho_{s} / \rho \text { is the } s \text {-th species mass fraction. }
$$

\subsection{Generalities on RD schemes ${ }^{1}$}

Solving System (1) by means of $R D$ schemes requires expanding the solution on the $P 1$ finite element basis associated to a simplicial tessellation $\Omega^{h}$ ( $n_{E \text { tem }}$ elements and $n_{D \rho F}$ nodes), i.e., $\bar{U}^{h}(\bar{x}, t)=\sum_{j=1}^{N_{D O F}} \bar{U}_{j}(t) N_{j}(\vec{x})$. The discrete equation governing the evolution of the 1 -th DoF is:

$$
v_{\Xi_{1}} \frac{d \bar{U}_{1}}{d t}+\bar{\Phi}_{1}=\overline{0}
$$

where $\vec{\Phi}_{l}$ is the steady state nodal residual, that gathers convective and source contributions, i.e., $\vec{\Phi}_{i}=\bar{\Phi}_{i}^{c v}-\bar{\Phi}_{i}^{5}$. This nodal residual is assembled from contributions from the set $\Xi_{l}$ of all the elements including node $l$, as in $\vec{\Phi}_{i}=\sum_{\Omega_{e} \in \Xi_{i}}\left(\bar{\Phi}_{i}^{c v, \Omega_{e}}-\bar{\Phi}_{i}^{S, \Omega_{e}}\right)$. A specific RD scheme is defined then by how elemental residuals $\vec{\Phi}^{\Omega_{e}}$ are transferred to $l$-th node. An elemental residual is:

$$
\vec{\Phi}^{\Omega_{e}}=\int_{\Omega_{e}} \frac{\partial \bar{F}_{j}^{c v}}{\partial x_{j}} d v-\int_{\Omega_{e}} \vec{S} d v=\vec{\Phi}^{c v \cdot \Omega}-\bar{\Phi}^{S, \Omega} .
$$

Focusing now on the convective residual, it is given by:

$$
\vec{\Phi}_{i}^{c \nu}=\sum_{\Omega_{e}=\Xi_{l}} \vec{\Phi}_{i}^{\text {cv. } \Omega_{e}}\left(K_{1}^{\Omega_{e}} \ldots, K_{n_{D}+1}^{\Omega_{2}}\right)
$$

where we explicitly state that the element-to-node residual distribution is formulated in terms of the upwind parameters $K_{j}^{\Omega_{e}}=\frac{1}{n_{D}} A_{\dot{x}_{d}}^{c \nu, \Omega_{e}}\left(\vec{U}_{\text {avg }}\right) n_{x_{d}}^{\Omega_{e}} ; \vec{U}_{\text {avg }}$ is a cell-averaged state and $n_{x_{d}}$ are the components of inward scaled normals and Einstein convention applies again.

$R D$ schemes can be classified according to how the discrete convective residual is computed: we distinguish among linearization-based (LRD) and contour-integration-based (CRD) schemes. The distinction lies on whether one can find a variable set $\vec{Z}$ such that both the unknown $\vec{U}$ and the flux vector $\vec{F}_{j}^{c v}$ are quadratic functions of the components of $\bar{Z}$. In that case, by linearizing $\vec{F}_{j}^{c v}$ we obtain $\vec{\Phi}^{c \nu, \Omega_{e}}=\int_{\Omega_{e}} \frac{\partial \vec{F}_{j}^{c v}}{\partial x_{j}} d v=\int_{\Omega_{e}} \frac{\partial \vec{F}_{j}^{c v}}{\partial \vec{Z}} \cdot \frac{\partial \vec{Z}}{\partial x_{j}} d v$. Since the solution is expressed in $P 1$ (linear) elements, $\left.\frac{\partial^{\mathrm{h}} \bar{Z}}{\partial x_{j}}\right|_{\Omega_{e}}$ is constant. At the same time, the Jacobian $\frac{\partial \bar{F}_{j}^{v}}{\partial \bar{Z}}$ is a linear function and henceforth the following relation:

$$
\vec{\Phi}^{c v, \Omega_{e}}=\int_{\Omega_{e}} \frac{\partial \vec{F}_{j}^{c v}}{\partial \vec{Z}} d v=V_{\Omega_{e}} \frac{\partial \bar{F}_{j}^{c v}}{\partial \bar{Z}}\left(\vec{U}\left(\vec{Z}_{a v g}\right)\right)
$$

holds exactly. The exact value of $\vec{\Phi}^{c v, \Omega_{e}}$ is therefore given by the simple relation $\vec{\Phi}^{c v, \Omega_{e}}=\left.V_{\Omega_{e}} \frac{\partial \vec{F}_{j}^{c \nu}}{\partial \grave{Z}}\left(\bar{U}\left(\vec{Z}_{a v g}\right)\right) \frac{g^{h t} \tilde{Z}}{\partial x_{j}}\right|_{\Omega_{e}}$.

Whenever a $\vec{Z}$ variable is not readily available, one can resort to CRD schemes, by evaluating numerically the element residual $\vec{\Phi}^{c, \Omega_{e}}$ via Gauss quadrature:

$$
\vec{\Phi}^{c, \Omega_{e}}=\int_{\Omega_{e}} \nabla \cdot \vec{F}_{j}^{c v} d v=\oint_{\delta \Omega_{e}} \bar{F}_{j}^{c v} \cdot \bar{e}_{j}^{e x t} d s=\sum_{f=1}^{n_{D}+1} \int_{S_{j}} \vec{F}_{j}^{c v} \cdot \vec{e}_{j}^{e x t} d s=\sum_{f=1}^{n_{D}+1} \sum_{q=Q} \omega_{q}\left(\bar{F}_{j}^{c v} \cdot \bar{e}_{j}^{e x t}\right)_{q} l_{q} .
$$

The only restriction is that the quadrature error is lower than that of the distribution error.

\footnotetext{
1 The interested reader will find extensive detaiks on the $R D$ discretization for the system of equations (1) in references $[5,13,14]$
} 
In this work we only consider the LRD N scheme [5,13] and its CRD counterpart, the Nc scheme [15]. The $N$ scheme contribution to the nodal residual is given by:

$$
\vec{\Phi}_{i}^{c . \Omega_{e} N}=K_{i}^{+} \cdot\left(\bar{U}_{l}-\vec{U}_{\text {intet }}^{\Omega_{e}}\right) \text { with } \bar{U}_{\text {intet }}^{\Omega_{e}}=\left(\sum_{j \in \Omega_{e}} K_{j}^{-}\right)^{-1} \cdot \sum_{j \in \Omega_{e}} K_{j}^{-} \cdot \bar{U}_{j} .
$$

Concerning the CRD variant, the $N_{c}$ scheme is given by $\bar{\Phi}_{i}^{c, \Omega_{e} . N_{c}}=\vec{\Phi}_{i, *}^{c \Omega_{e} . N}-B_{i}^{\Omega_{e}, L D A} \delta \vec{\Phi}^{c}$, where $\bar{\Phi}_{i, *}^{c \Omega_{p} . N}$ is the result of the inconsistent evaluation of $\mathrm{Eq} .(10)$ and $\delta \bar{\Phi}^{c}$ is a conservation correction given by:

$$
\delta \vec{\Phi}^{c}=\left.\vec{\Phi}_{\Omega, *}^{c}\right|_{\text {Inconsistent }}-\left.\bar{\Phi}_{\Omega}^{c}\right|_{\text {Gauss }}=\sum_{j \in \Omega_{e}} K_{j} \vec{U}_{j}-\left.\bar{\Phi}_{S \Omega}^{c}\right|_{\text {Gouss }} .
$$

A relevant difference between the $N$ scheme and its CRD variant which is specially relevant for this work is that the Nc scheme is non-positive, meaning that the capture of certain shock waves may present oscillations. This is not a concern for perfect gas simulations, as long as one uses the Roe-Struijs-Deconinck linearization variable $\bar{Z}$ [16] (in that case Nc and $N$ schemes coincide). The lack of monotonicity is, however, specially problematic for NEQ flows whenever strong, bow shock waves are present in the domain; this is precisely the case when the $\vec{Z}$ variable is less likely to be well-defined, see section 4). The underlying reason is the mass production terms $-\dot{\omega}_{s}$ - highly non-linear dependence on temperature: the extreme variations in temperature across the numerically captured shock wave result in unphysical production/destruction of species, and this leads eventually to simulation blow up [7].

\section{Description of the multi-dimensional Roe-Liu-Vinokur linearization procedure}

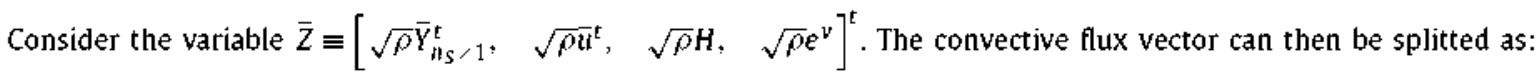

$$
\bar{F}_{j}^{c v}=\underbrace{\bar{F}_{j}^{c v}-\left[\overrightarrow{0}_{n_{s \times 1}}, p \overline{1}_{j}, 0,0\right]^{t}}_{\vec{Q}}+\underbrace{\left[\overline{0}_{n_{s} \times 1}, p \overline{1}_{j}, 0,0\right]^{t}}_{\vec{\pi}} .
$$

All terms in $\vec{Q}$ are quadratic functions of the components of $\vec{Z}$, and thus entries in $\frac{\partial \vec{F}_{j}^{c v}}{\partial \vec{Z}}$ are simply linear functions; pressure is isolated in $\vec{\Pi}$. Its differential is given by Eq. (4); in a multi-dimensional upwind framework, we rather consider relation Eq. (4) at the gradient level:

$$
\nabla p=\sum_{s=1}^{n_{S}} \gamma_{s} \nabla \rho_{s}+\beta \nabla \rho e^{t r}
$$

which can be immediately recast in terms of the components of parameter vector $\bar{Z}$ by using the relation $\rho e^{t r}=$ $\rho H-\frac{\rho \vec{u}^{t} \vec{u}}{2}-\rho e^{v}-p$, and considering $\rho_{s}=\left(\sum_{r=1}^{n_{s}} \sqrt{\rho} y_{r}\right) \sqrt{\rho} y_{s}, \rho \bar{u}^{t} \cdot \vec{u}=\sqrt{\rho} \bar{u}^{t} \cdot \sqrt{\rho} \vec{u}, \rho H=\left(\sum_{r=1}^{n_{s}} \sqrt{\rho} y_{r}\right) \sqrt{\rho} H_{\text {, and }}$ $\rho e^{v}=\left(\sum_{r=1}^{n_{s}} \sqrt{\rho} y_{r}\right) \sqrt{\rho} e^{v}$.

At this point, if one managed to find values for $\gamma_{s}$ and $\beta$ for which Eq. (13) holds, the gradient of pressure would be then be defined in terms of $\bar{Z}$, making in turn $\frac{\partial \bar{n}}{\partial \bar{Z}}$ a linear function of the parameter vector as well. In that case, evaluating $A_{j}^{c . U}$ at an averaged state $\bar{Z}_{\text {avg }}$ would provide a conservative convective residual. Unfortunately. Eq. (13) provides only as many equations as the dimensionality $n_{D}$ of the problem (namely 1,2 or 3 ) while $n_{S}+1$ parameters have to be defined for the case of a non-ionized $n_{5}$ species gas mixture.

In order to determine these $n_{S}+1$ parameters, we can interpret Eq. (13) as $n_{D}$ restrictions $^{2}$ :

$$
r_{j} \equiv \frac{\partial p}{\partial x_{j}}-\sum_{s=1}^{n_{s}} \frac{\partial \rho_{s}}{\partial x_{j}} \gamma_{s}-\frac{\partial \rho e^{t r}}{\partial x_{j}} \beta=0, j=1 \ldots n_{0},
$$

that the solution sought should respect in order to guarantee conservation. In that case, it would be sensible to look for a solution which, while fulfilling the aforementioned restrictions (guaranteeing thus conservation), is the closest one to a given a priori approximation, like for example the nodal averages $\hat{\gamma}_{s}=\sum_{i=1}^{n_{D}+1} \frac{\left.\gamma_{s}\right|_{1}}{n_{D}+1}$ and $\hat{\beta}=\sum_{i=1}^{n_{D}+1} \frac{\left.\beta\right|_{1}}{n_{D}+1}$.

\footnotetext{
2 Here, the $\frac{\partial}{\partial x_{j}}$ operator applied to pressure is the discrete FE one $\frac{\partial^{h} g}{\partial x_{j}}=\frac{1}{2 v_{a_{k}}} \sum_{k=1}^{n_{0}+1} p_{k} n_{j-k}$. The same operator applied to any other magnitude in should

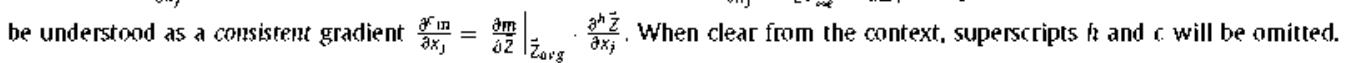




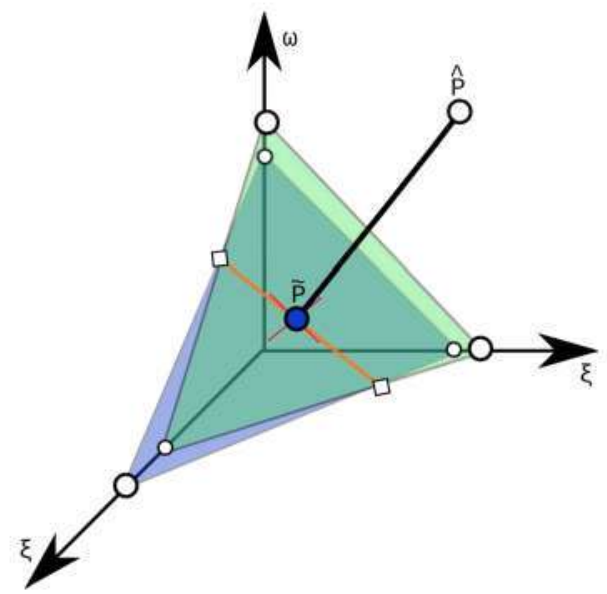

Fig. 1. Constrained minimization problem: graphical interpretation. (For interpretation of the colors in the figure(s), the reader is referred to the web version of this article.)

Under these hypotheses, we are faced with a constrained minimization problem. According to [1], in order not to obtain a solution dependent on the arbitrary reference enthalpy entering in the definition of the parameters $\gamma_{s}$, it is better to work on the space $\left\{\xi_{1}, \ldots, \xi_{N} ; \omega\right\}$, where $\xi_{s}=\gamma_{s} / \beta, s=1, \ldots, n_{S}$ and $\omega=1 / \beta$.

The Lagrangian for the restricted minimum distance problem is:

$$
\mathcal{L} \equiv(\omega-\hat{\omega})^{2}+\frac{1}{\hat{\sigma}^{2}} \sum_{s=1}^{n_{S}}\left(\xi_{s}-\hat{\xi}_{s}\right)^{2}-\vec{\lambda}^{t} \cdot\left(\nabla p \omega-\sum_{s=1}^{n_{S}} \nabla \rho_{s} \xi_{s}-\nabla \rho e^{t r}\right) .
$$

Factor $\hat{\sigma}^{-2}$ is included for dimensional consistency, and $\hat{\sigma}$ taken as the local speed of sound squared. The corresponding stationarity conditions are:

$$
\begin{aligned}
& \frac{\partial \mathcal{L}}{\partial \xi_{r}}=\frac{2}{\hat{\sigma}^{2}}\left(\xi_{r}-\hat{\xi}_{r}\right)+\vec{\lambda}^{t} \cdot \nabla \rho_{r}=0, r=1 \ldots n_{S}, \quad \frac{\partial \mathcal{L}}{\partial \omega}=2(\omega-\hat{\omega})-\vec{\lambda}^{t} \cdot \nabla p=0, \\
& \frac{\partial \mathcal{L}}{\partial \lambda_{j}}=\frac{\partial p}{\partial x_{j}} \omega-\sum_{s=1}^{n_{S}} \frac{\partial \rho_{s}}{\partial x_{j}} \xi_{s}-\frac{\partial \rho e^{t r}}{\partial x_{j}}, j=1 \ldots n_{D} .
\end{aligned}
$$

Fig. 1 shows the graphical interpretation of the minimization problem defined by the Lagrangian in Eq. (15) for a $2 D$ computation with the $\mathrm{N}-\mathrm{N}_{2}$ mixture $\left(n_{s}=2\right)$ ) starting from an approximation $\hat{P}$ in the space $\left\{\xi_{r} ; \omega\right\}$, we look for the closest point $\tilde{P}$ which fulfills at the same time the $n_{D}=2$ restrictions, that is, the point that lies on the line where both the blue and the green planes intersect.

The stationarity conditions Eq. (16) translate into a linear system of equations $\mathcal{A} \cdot \vec{x}=\vec{b}$. The coefficient matrix $\mathcal{A}$ is, after application of elemental operations:

$$
\mathcal{A}=\left[\begin{array}{c|c|ccc}
\overline{\bar{I}}_{n_{S}} & 0 & \frac{\hat{\sigma}^{2}}{2} \frac{\partial \rho \vec{Y}_{n_{S} \times 1}}{\partial x} & \frac{\hat{\sigma}^{2}}{2} \frac{\partial \rho \vec{Y}_{n_{S} \times 1}}{\partial y} & \frac{\hat{\sigma}^{2}}{2} \frac{\partial \rho \vec{Y}_{n_{S} \times 1}}{\partial z} \\
\hline \overrightarrow{0}_{1 \times n_{S}} & 1 & -\frac{1}{2} \frac{\partial p}{\partial x} & -\frac{1}{2} \frac{\partial p}{\partial y} & -\frac{1}{2} \frac{\partial p}{\partial z} \\
\hline \overrightarrow{0}_{1 \times n_{S}} & 0 & C_{x x} & C_{x y} & C_{x z} \\
\overrightarrow{0}_{1 \times n_{S}} & 0 & C_{x y} & C_{y y} & C_{y z} \\
\overrightarrow{0}_{1 \times n_{S}} & 0 & C_{x z} & C_{y z} & C_{z z}
\end{array}\right],
$$

the vector of unknowns $\vec{x}=\left[\xi_{1}, \ldots, \xi_{N} ; \omega ; \lambda_{x_{j}}\right]^{t}$ and of the forcing vector $\vec{b}=\left[\hat{\xi}_{1}, \ldots, \hat{\xi}_{N} ; \hat{\omega} ;-\hat{\omega} \delta p_{x_{j}}\right]^{t}$. In Eq. (17), $C_{x_{i} x_{j}}$ stands for:

$$
C_{x_{i} x_{j}}=\frac{1}{2}\left(\frac{\partial p}{\partial x_{i}} \frac{\partial p}{\partial x_{j}}\right)+\frac{\hat{\sigma}^{2}}{2} \sum_{s=1}^{n_{s}}\left(\frac{\partial \rho_{s}}{\partial x_{i}} \frac{\partial \rho_{s}}{\partial x_{j}}\right),
$$

while $-\hat{\omega} \delta p_{x_{j}}$ is:

$$
-\hat{\omega} \delta p_{x_{j}}=(1+\hat{\omega}) \frac{\partial p}{\partial x_{j}}-\sum_{S=1}^{n_{S}} \frac{\partial \rho_{s}}{\partial x_{j}} \hat{\xi}_{s}-\frac{\partial}{\partial x_{j}}\left(\rho H-\frac{1}{2} \rho \vec{u}^{t} \cdot \vec{u}-\rho e^{v}\right) .
$$


Testcase definition: NEQ two jets.

\begin{tabular}{lllllll}
\hline & $M a s$ & $p_{\infty}(\mathrm{kPa})$ & $T_{\infty}=T_{\infty}^{v}(\mathrm{~K})$ & $\rho_{\infty}\left(\mathrm{kg} / \mathrm{m}^{3}\right)$ & $y_{N_{3}}$ & $y_{N_{2}, \infty}$ \\
\hline$y<0.5$ & 2.4 & 10.3 & 1750 & 0.019824 & 0 & 1 \\
$y>0.5$ & 4.7 & 5 & 3500 & 0.004809 & $6 / 10^{-4}$ & 0.9994 \\
\hline
\end{tabular}

Observe how the structure of the linear system is such that a $n_{D} \times n_{D}$ subsystem:

$$
\mathcal{B} \bar{\lambda}=-\hat{\omega} \bar{\delta} \bar{p} .
$$

can be decoupled. Provided one can invert $\mathcal{B}, \xi_{s}$ and $\omega$ are immediately obtained by back substitution:

$$
\hat{\omega}=\hat{\omega}+\frac{1}{2}\left(\lambda_{x} \frac{\partial p}{\partial x}+\lambda_{y} \frac{\partial p}{\partial y}+\lambda_{z} \frac{\partial p}{\partial z}\right) \text {, and } \xi_{s}=\hat{\xi}_{s}-\frac{\hat{\sigma}^{2}}{2}\left(\lambda_{x} \frac{\partial \rho_{s}}{\partial x}+\lambda_{y} \frac{\partial \rho_{s}}{\partial y}+\lambda_{z} \frac{\partial \rho_{s}}{\partial z}\right) \text {. }
$$

The values actually employed in the computation are obtained in a straightforward manner from $\tilde{\beta}=\frac{1}{\bar{\theta}}$ and $\tilde{\gamma}_{s}=\tilde{\beta} \tilde{\xi}_{s}$.

Clearly, the success of the linearization process revolves around the invertibility of $\mathcal{B}$. A priori one would expect this task to be relatively straightforward, $\mathcal{B}$ being a real symmetric matrix. However, even for the most simple non-equilibrium two jets problems (see below), one obtains solutions $\bar{\beta}, \tilde{\gamma}_{s}$ that respect conservation at the discrete level (that is, with the restrictions $r_{j}$ equal to machine zero) but which correspond to unphysical situations (e.g. $\tilde{\beta}<0$ or $c^{2}<0$ ). Since there is a certain degree of freedom to determine the pressure derivatives, this unexpected difficulty can be handled easily by simply setting $\hat{\beta}=\hat{\beta}$ and determining the set of $\dot{\gamma}_{s}$ by the same minimization procedure described above. One simply needs to notice that the matrix coefficients are now $c_{x_{i} x_{j}}=\frac{\hat{i}^{2}}{2} \sum_{s=1}^{n_{S}}\left(\frac{\partial \rho_{s}}{\partial x_{1}} \frac{\partial \rho_{s}}{\partial x_{j}}\right)$.

[n this manner, we have been able to solve a $N E Q$-variant of the two jets problem in [17]. The problem consists in two uniform, supersonic $N-N_{2}$ streams discharging into a square domain. The conditions of each of the jets are given in Table 1 .

We have computed this testcase both with the LRD $N$ and the CRD NC schemes on the completely unstructured grid $\left(n_{\text {Etem }}=5638, n_{\text {Points }}=2920\right)$ obtained by Delaunay triangulation (see also Fig. $2(\mathrm{~b})$ ).

The results are summarized in Fig. 2. Despite the complex thermodynamic model in non-equilibrium considered, the solution presents the familiar shock-fan-contact structure, see Figs. 2(a)-2(b). Observe how no differences are apparent between the LRD and the CRD-based solutions, see Figs. 2(c)-2(d).

There is an important difference, however, in the effort needed to obtain each of the solutions. In both cases an initial condition consisting in an uniform flow field has been considered: on the one hand, the CRD solution needed to be initialized with an explicit pseudo-time stepping procedure; non-monotonicity of the CRD scheme resulted in temperature undershoots that were addressed by clipping temperature to $T_{\min }=200 \mathrm{~K}$ for $\approx 40000$ iterations with $C F L<0.1$. Once the shock was in place, the CRD solution could be restarted in implicit mode, but maintaining the clipping active almost until convergence was achieved. On the other hand, the LRD solution converged easily to steady state in less than 250 implicit iterations, starting from the uniform flow field; $T$-clipping was never needed.

Therefore, in view of the non-monotonicity of the CRD Nc scheme, it seems that one should favor the LRD $N$ scheme. Unfortunately, when addressing slightly more involved testcases, e.g. an inviscid NEQ ramp flow, we observed how the very same LRD $N$ scheme was incapable of providing a converged solution; actually and more often than not the simulation blew up. A similar behavior was reported in [18]. Later on Pepe et al. [19] succeeded in applying the multi-dimensiona] Roe-Liu-Vinokur linearization to a quasi-neutral argon plasma flow in thermal equilibrium; it might seem that the fact of the plasma is formed exclusively by monoatomic species is important for the multi-dimensional RLV procedure to work. In the next section we investigate this behavior by focusing on the well-posedness of the linear system (20).

\section{On the well-posedness of the multi-dimensional Roe-Liu-Vinokur linearization for TCNEQ flows}

In previous section we pointed out that the success of the linearization procedure hinges on the invertibility of submatrix $\mathcal{B}$ in Eq. (20), which is symmetric and with real entries. Such matrices are possibly the most favorable case one could expect for ... assuming that the determinant of the matrix is not zero, of course!

The analysis of the invertibility of $\mathcal{B}$ is greatly eased by introducing matrix $M$, given by:

$$
M \equiv\left[\begin{array}{cccc}
\frac{\partial^{c}}{\partial x} \rho_{1} & \cdots & \frac{\partial^{c}}{\partial x} \rho_{n_{S}} & \frac{\partial^{\mathrm{h}}}{\partial x} p \\
\frac{\partial^{c}}{\partial y} \rho_{1} & \cdots & \frac{\partial^{c}}{\partial y} \rho_{n_{S}} & \frac{\partial^{\mathrm{h}}}{\partial y} p \\
\frac{\partial^{c}}{\partial z} \rho_{1} & \cdots & \frac{\partial^{c}}{\partial z} \rho_{n_{S}} & \frac{\partial^{\mathrm{h}}}{\partial z} p
\end{array}\right] \in M_{n_{D} \backslash N} \text { with } N=n_{S}+1 .
$$

The whole minimization problem in $\mathrm{Eq}$. (20) can be expressed then in terms of matrix $M$, as:

$$
M \cdot M^{t} \cdot \vec{\lambda}=\vec{r}
$$




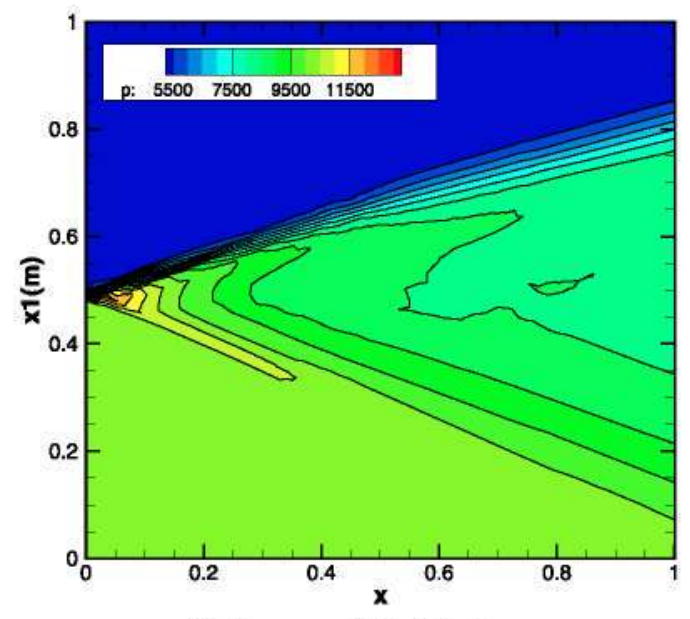

(a) Pressure $(\mathrm{Pa}), \mathrm{N}$ scheme.

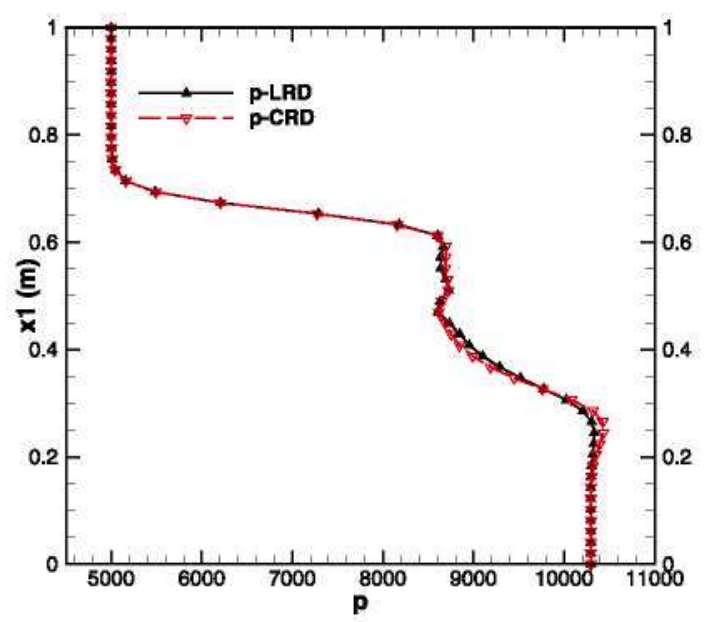

(c) Pressure (Pa).

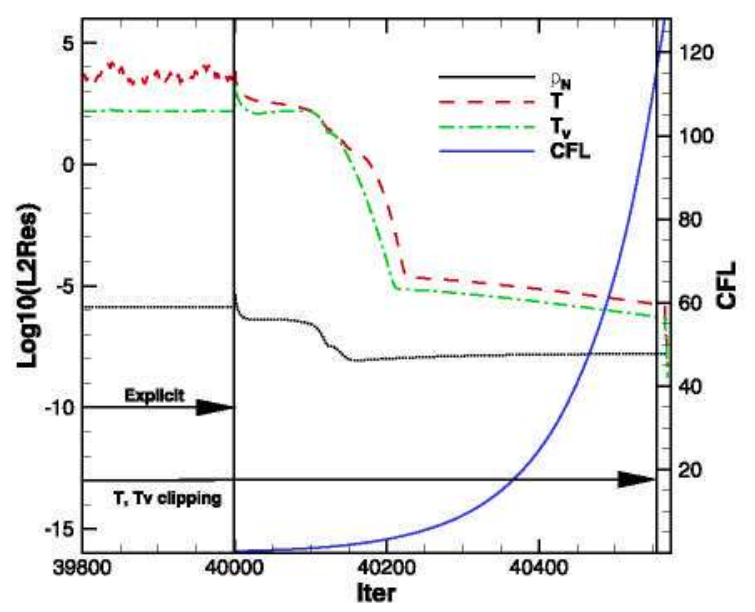

(e) $C R D$ simulation.

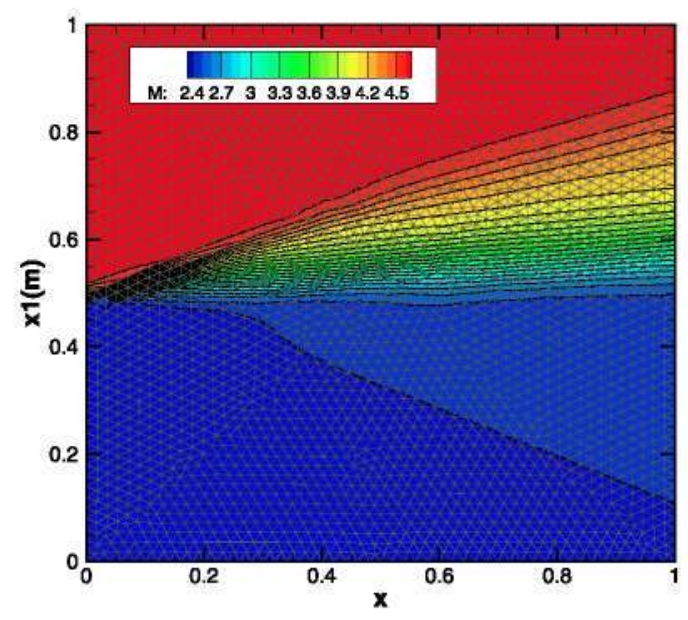

(b) $M a$ number, $N$ scheme.

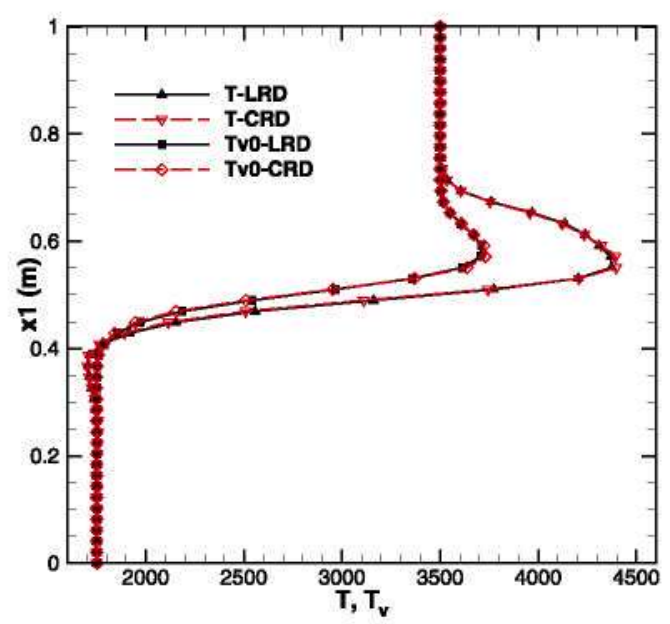

(d) $T$ and $T v(K)$.

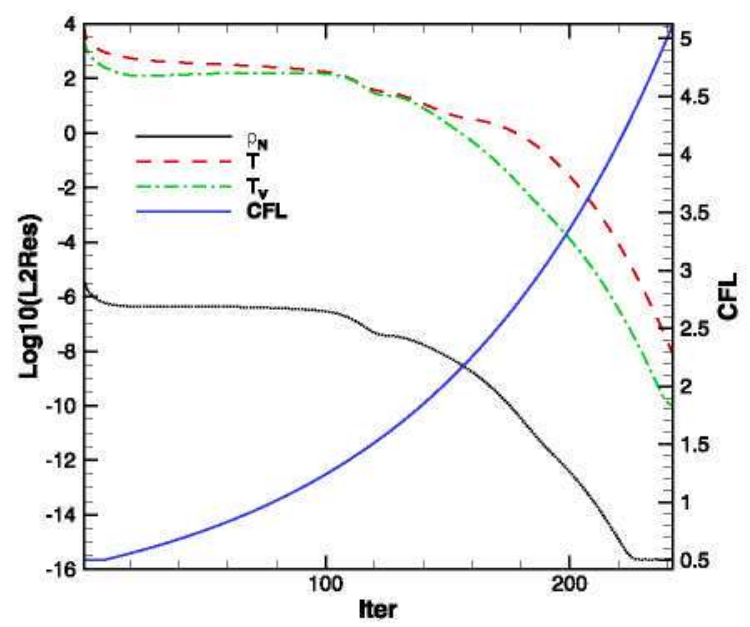

(f) $L R D$ simulation.

Fig. 2. Two jets NEQ problem. 
Since the rhs $\bar{r}=(1+\hat{\omega}) \nabla^{h} p-\sum_{s=1}^{n_{S}} \hat{\xi}_{s} \nabla^{c} \rho_{s}-\nabla^{c}\left(\rho H-\rho e_{v}-\frac{\rho u^{2}}{2}\right)$, matrix $M$ also concurs in the definition of $r$ :

$$
\overrightarrow{\mathrm{r}}=M \cdot\left[\begin{array}{c}
-\hat{\xi}_{1} \\
\cdots \\
-\hat{\xi}_{n_{s}} \\
1+\hat{\omega}
\end{array}\right]-\nabla^{c}\left(\rho H-\frac{1}{2} \rho \overline{\mathbf{r}}^{t} \cdot \vec{u}-\rho e^{v}\right) \equiv M \cdot \overline{\mathbf{r}}_{1}+\overrightarrow{\mathrm{r}}_{2} .
$$

Consider now a situation where the gradients of all species are aligned, i.e. across a normal shock wave or a contact discontinuity. In such a case:

$$
\nabla^{c} \rho_{2}=\alpha_{2} \nabla^{c} \rho_{1}, \quad \ldots, \quad \nabla^{c} \rho_{n S}=\alpha_{N-1} \nabla^{c} \rho_{1}, \quad \nabla^{h} p=\alpha_{N} \nabla^{c} \rho_{1}, \quad \text { and } \nabla^{c}\left(\rho H-\rho e_{v}-\frac{\rho u^{2}}{2}\right)=\alpha_{N+1} \nabla^{c} \rho_{1} ;
$$

the coefficient matrix $\mathcal{B}$ reduces then to:

$$
\mathcal{B}=M \cdot M^{t}=\left(1+\alpha_{2}^{2}+\ldots+\alpha_{N}^{2}\right)\left[\begin{array}{l}
m_{x, 1} \\
m_{y, 1} \\
m_{z, 1}
\end{array}\right] \cdot\left[m_{x, 1} m_{y, 1} m_{z, 1}\right]=\left(1+a^{2}\right) \bar{m}_{1} \cdot \vec{m}_{1}^{t}
$$

Notice how the determinant of $\vec{m}_{1} \cdot \vec{m}_{1}^{t}$ is zero. The rhs becomes in tum:

$$
\overline{\mathrm{r}}=\left(1+\hat{\omega}-\sum_{s=1}^{n_{S}} \hat{\xi}_{s}-\alpha_{N}\right) \vec{m}_{1}=b \bar{m}_{1} .
$$

The linear system is hence $\bar{m}_{1}\left(\left(1+a^{2}\right) \vec{m}_{1}^{t} \cdot \vec{\lambda}-b\right)=0$.

Therefore, we have shown that matrix $\mathcal{B}$ determinant is zero whenever the gradients of all the quantities $\left(\rho_{s}, p, \ldots\right)$ are aligned: that is, whenever there is a normal shock wave or a contact on the domain, the linearization procedure leads to a situation where there might be either an infinite number of solutions, or no solution at all.

The matter is further complicated if we take into account round-off errors: determinant $|\mathcal{B}|$ could be not zero but very small and the linearization procedure being extremely ill-conditioned.

In an attempt to alleviate the conditioning problem, we considered a truncated SVD technique. Matrix $\mathcal{B}$ is expressed as the product:

$$
\mathcal{B} \rightarrow L \cdot S \cdot R^{t}
$$

Matrices $L, R \in \mathbb{F}^{n_{D} / n_{D}}$ are orthogonal, and $S \in \mathbb{R}^{n n_{D}} \backslash n_{D}$ is the singular values matrix. Substituting into Eq. (23):

$$
L \cdot S \cdot R^{t} \cdot \bar{\lambda}=-\hat{\alpha} \delta \bar{p} \text {. }
$$

Pre-multiplying by $L^{t}$ leads to:

$$
S \cdot R^{t} \cdot \vec{\lambda}=-\hat{\omega} \overline{\delta p} \text {. }
$$

For the aligned-gradient case we know that at least one of the diagonal entries is zero (or very close to machine zero). We can obtain a regularized solution $\vec{\lambda}^{*}$ to (30):

$$
\vec{\lambda}^{*}=-R \cdot S^{+} \cdot L^{t} \cdot \hat{\omega} \delta \bar{p} .
$$

where the diagonal matrix $S^{+}$contains either the inverse of the eigenvalues/singular values of $S$ (i.e., $\frac{1}{S_{1}}$ ) or zero (if $s_{i}<\varepsilon$ ), with $\varepsilon$ a threshold parameter to decide when to neglect a particular singular value.

In our experience, the truncated SVD inversion does not, unfortunately, prevent the ill-posedness problems; when considering situations more complex than the two jets testcase of section 3, we were incapable of identifying a value of the threshold parameter $\varepsilon$ that made the linearizer perform properly. One should bear in mind that the choice of $\varepsilon$ affects with which level of accuracy the conservation restrictions Eq. (14) are fulfilled.

Alternatively, we attempted to solve Tikhonov-regularized normal equations: $\left(\mathcal{B}^{t} \cdot \mathcal{B}+\nu \overline{I_{n_{D}}}\right) \cdot \vec{\lambda}=-\mathcal{B}^{t} \cdot \hat{\omega} \vec{p}$; we observed again how, for those values of $v$ capable of regularizing the solution obtained was not conservative.

In conclusion, the multi-dimensional Roe-Liu-Vinokur linearization is not robust enough to compute shocked hypersonic flow fields. It is not clear whether more numerically reliable (pseudo-)inversion techniques could bring any advantage; this, however, remains beyond the scope of this work. Meanwhile, an alternative to address such problems was derived in [7], consisting in regularizing standard contour-integration-based RD schemes. Yet another alternative potentially worth to explore is considering the multi-dimensional extension of the approach described in [20,21]. 


\section{Conclusions}

In this short note we have described the lack of robustness afflicting the standard formulation of the multi-dimensional Roe-Liu-Vinokur linearization of [22] when used with linearization-based residual distribution (RD) schemes. By focusing on the linear system associated to the linearization procedure we have identified situations where such system is ill-posed. We have seen how standard regularization techniques (e.g., truncated SVD or Tikhonov-regularized normal equations) do not suffice to retrieve a linearized state that guarantees conservation at the discrete level.

Since standard contour-integration-based $R D$ schemes are non-monotonic, does that mean that is not possible to compute hypersonic non-equilibrium flow fields using RD schemes? Fortunately, that is not the case. As we demonstrated recently in [7], by supplementing standard CRD schemes with a locally active shock capturing term it is possible to obtain solutions that are free from temperature under/overshoots across the shock-captured shock wave.

By no means we claim that the regularized CRD schemes in [7] are the only possible strategy to address non-equilibrium hypersonic flows. Actually, as long as it is possible to fit all the shock structures present in the computational domain, then the optimal approach would be to use 2nd-order standard CRD (not regularized) schemes in combination with the shock fitting strategies in [23-25].

However, whenever a shock front cannot be properly fitted, the regularized CRD schemes are instrumental to obtain converged solutions. Research is undergoing to improve the regularization techniques for RD schemes: e.g. [26] describes an enthalpy-preserving shock capturing term which can be extended in a straightforward manner to the complex thermochemical non-equilibrium model considered in this work. Alternative, supplementary dissipative terms are described in [27, 28].

Finally, note that alternatives to the Liu and Vinokur Roe-like linearization exist in the literature. One of those might well be the approach described in [20,21]: it is worth considering whether achieving a multi-dimensional extension is possible.

\section{Acknowledgements}

First author has been supported by a Belgian FRIA fellowship from the Fonds National de la Recherche Scientifique and by the European Commission Research and Innovation action DRAGY (Grant Agreement 690623).

The authors thankfully acknowledge the computer resources, technical expertise and assistance provided by the Supercomputing and Visualization Center of Madrid (CeSViMa).

\section{References}

[1] Y. Liu, M. Vinokur, Upwind algorithms for general thermo-chemical nonequilibrium flows, in: Proceedings of the 27th AlAA Aerospace Science Meeting and Exhibit, AIAA, Reno (NV), 1989.

[2] P. Roe, Approximate Riemann solvers, parameter vectors, and difference schemes, J. Comput. Phys. 43 (1981) 357-372.

[3] N. Tsuboi, A. Hayashi, Y. Matsumoto, Three-dimensional parallel simulation of cornstarch-oxygen two-phase detonation, Shock Waves 10 (2000) $277-285$.

[4] N. Tsuboi, M. Asahara, K. Eto, A.K. Hayashi, Numerical simulation of spinning detonation in square tube, Shock Waves 18 (2008) $329-344$.

[5] E. van der Weide, Compressible Flow Simulation on Unstructured Grids Using Multi-Dimensional Upwind Schemes, Ph.D. thesis, TU Delft, 1998.

[6] J. Garicano-Mena, P. Solano-López, G. Degrez, On the multidimensional roe-like linearization for non-equilibrium multi-species gas mixtures: applica tion to residual distribution schemes, in: 7th European Conference on Computational Fluid Dynamics, 2018.

[7] J. Garicano-Mena, A. Lani, G. Degrez, An entropy-variables-based formulation of residual distribution schemes for non-equilibrium flows, J. Comput. Phys, 362 (2018) 163-189.

[8] P.A. Gnoffo, Upwind-biased, point-implicit relaxation strategies for viscous, hypersonic flows, in: Proceedings of the 37th AIAA Aerospace Science Meeting and Exhibit, AIAA, Portland (OR), 2004.

[9] A. Chorin, J.E. Marsden, A Mathematical Introduction to Fluid Mechanics, 3rd edition, Springer, 2000.

[10] C Hirsch, Numerical Computation of Internal and External Flows: Introduction to the Fundamentals of CFD, 2nd edition, Butterworth-Heinemann, 2006.

[11] P. Gnoffo, R. Gupta, J. Shinn, Conservation Equations and Physical Models for Hypersonic Air Flows in Thermal and Chemical Non-equilibrium, TP 2867 NASA, 1989

[12] M. Panesi, Physical Models for Nonequilibrium Plasma Flow, Ph.D. thesis, Università degli studi di Pisa, 2009.

[13] R. Abgrall, Toward the ultimate conservative scheme: following the quest, J. Comput. Phys. 167 (2001) 277-315.

[14] N. Villedieu, T. Quintino, M. Ricchiuto, H. Deconinck, Third order residual distribution schemes for the Navier-Stokes equations, J. Comput. Phys. 230 (2011) 4301-4315, Special issue High Order Methods for CFD Problems.

[15] A. Csik, M. Ricchiuto, H. Deconinck, Conservative formulation of the multidimensional upwind residual distribution schemes for general nonlinear conservation laws, J. Comput. Phys. 179 (2002) 286-312.

[16] H. Deconinck, P. Roe, R. Struijs, A multidimensional generalization of Roe's flux difference splitter for the Euler equations, Comput. Fluids 22 (1993) $215-222$.

[17] H.M. Glaz, A.B. Wardlaw, A high-order Godunov scheme for steady supersonic gas dynamics, J. Comput. Phys. 58 (1985) $157-187$.

[18] R. Pepe, Private communication, 2012.

[19] R. Pepe, A. Bonfiglioli, A. D'Angola, G. Colonna, R. Paciorri, An unstructured shock-fitting solver for hypersonic plasma flows in chemical nonequilibrium, Comput. Phys, Commun. 196 (2015) 179-193.

[20] R. Abgrall, Preliminary Results on the Extension of Roe's Riemann Solver to Nonequilibrium Flows, Rapports de Recherche, vol, 987, INRIA, 1989.

[21] R. Abgrall, An extension of Roe's upwind scheme to algebraic equilibrium real gas models, Comput. Fluids 19 (1991) 171-182.

[22] G. Degrez, E, van der Weide, Upwind Residual Distribution Schemes for Chemical Non-equilibrium Flows, Collection of Technical Papers, vol. 2, AlAA, 1999, pp. 978-987.

[23] R. Paciorri, A. Bonfiglioli, A shock-fitting technique for 2D unstructured grids, Comput. Fluids 38 (2009) 715-726. 
[24] A. Bonfiglioli, M. Grottadaurea, R. Paciorri, E. Sabetta, An unstructured, three-dimensional, shock-fitting solver for hypersonic flows, Comput. Fluids 73 (2013) 162-174.

[25] A. Lani, V. De Amicis, SF: an open source object-oriented platform for unstructured shock-fitting methods, in; M, Onofri, R. Paciorri (Eds.), Shock Fitting. Shock Wave and High Pressure Phenomend, 2017.

[26] J. Garicano-Mena, G. Degrez, An enthalpy-preserving shock-capturing term for residual distribution schemes, Int. J. Numer. Methods Fluids 88 (2018) 385-411.

[27] R. Abgrall, D.D. Santis, Linear and non-linear high order accurate residual distribution schemes for the discretization of the steady compressible Navier-Stokes equations, J. Comput. Phys. 283 (2015) 329-359.

[28] R. Abgrall, P. Bacigaluppi, S. Tokareva, A high-order nonconservative approach for hyperbolic equations in fluid dynamics, Comput. Fluids 169 (2018) $10-22$. 